\title{
INTEGRACIÓN DE LOS CURSOS SPOC EN LAS ASIGNATURAS DE
} GRADO. UNA EXPERIENCIA PRÁCTICA

\section{INTEGRATION OF SPOC COURSES IN THE SUBJECTS OF DEGREE. A PRACTICAL EXPERIENCE}

\author{
Dra. Arantzazu López de la Serna \\ arantzazu.lopez@ehu.eus \\ Dr. Carlos Castaño Garrido \\ carlos.castano@ehu.eus \\ Dr. David Herrero Fernández \\ david.herrero@ehu.eus
}

\author{
Universidad del País Vasco/Euskal Herriko Unibertsitatea \\ Escuela Universitaria de Magisterio de Bilbao/Bilboko Irakasleen Magisteritza Eskola \\ Barrio Sarriena s/n 48940 Leioa. Bizkaia (España)
}

Los cursos SPOC (del inglés Small Private Online Course) son cursos deformación a distancia con una metodología participativa y colaborativa, características que comparten con los MOOC, aunque en este caso, los SPOC están destinados a grupos reducidos de estudiantes de perfil bien definido. La combinación de la enseñanza virtual y la enseñanza presencial es un recurso positivo para la comunidad universitaria que es importante analizar. En este estudio se elabora una comparación de dos grupos de estudiantes universitarios que realizan dos experiencias de aprendizaje, desarrolladas en contextos de aprendizaje muy diferentes, uno online a través de un curso SPOC insertado en una asignatura de Grado y otro presencial, en la que el alumnado hace el mismo curso pero dentro del contexto del aula.

Palabras clave: SPOC. Aprendizaje Autorregulado. Motivación. Enseñanza Superior. Asignaturas de Grado.

The SPOC (Small Private Online Course) courses are distance learning courses with a participatory and collaborative methodology, characteristics that they share with the MOOCs, although in this case, the SPOCs are aimed at small groups of well-defined students. The combination of virtual teaching and face-to-face teaching is a positive resource for the university community that is important to analyze. This study compiles a comparison of two groups of university students who carry out two learning experiences, developed in very different learning contexts, one online through an SPOC course inserted in a subject of Degree and one face-to-face, in which the Students do the same course but within the context of the classroom.

Key Words: SPOC. Self-regulated learning. Motivation .Higher education. Grade subjects.

- 139 -

Píxel-Bit. Revista de Medios y Educación. No 52 Enero 2018. ISSN: 1133-8482. e-ISSN: 2171-7966. doi: http://dx.doi.org/10.12795/pixelbit.2018.i52.10 


\section{Introducción.}

La aparición de los cursos MOOC en el contexto universitario ha repercutido de forma significativa en la universidad. Es en el año 2001 cuando el MIT desarrolla un proyecto en el que permite acceder de forma gratuita, a diferentes recursos educativos de su universidad. En pocos años, concretamente en el año 2008 fue cuando se publicó el primer curso considerado como el primer MOOC de la historia .Este curso titulado «Connectivism and Connective Knowledge» fue realizado por George Siemens y Stephen Downes, en la Universidad de Manitoba (Canadá). Los primeros cursos MOOC fueron diseñados en base a principios conectivistas, a través de los cuales se pretendía fomentar la filosofía surgida de los Recursos Educativos Abiertos (REA), donde principalmente se pretende reutilizar, colaborar y compartir todo tipo de contenidos dentro de un entorno completamente libre en el que, cualquier persona desde cualquier lugar pueda acceder. A través de los REA, millones de personas en todo el mundo podrán tener acceso a informaciones que pueden ser utilizadas para el desarrollo social y económico. (UNESCO, 2012). Los cursos MOOC han ido implantándose de forma destacada en las diferentes universidades del mundo, a través del estudio realizado en el año 2013 por (Cátedra Telefónica, 2014) casi un tercio de las universidades españolas han ofertado este tipo de formación durante el año 2013. Esto nos muestra que se están produciendo cambios desde hace tiempo dentro de las universidades que repercuten en la docencia, por lo que el profesorado debe ir adecuándose, junto con el alumnado a estas nuevas metodologías (Ghemawat, 2017). La educación superior se está convirtiendo en una actividad global y parece que la tendencia va a ir creciendo (Aguaded, Vázquez-Cano \& Sevillano, 2013; Vizoso Martín, 2013).La inserción en la universidad de nuevas modalidades pedagógicas colaborativas hacen que se fomenten formatos educativos que autores como, Vázquez-Cano (2013),señalan que en estos nuevos escenarios formativos universitarios se estén orientando hacia un nuevo modelo de formación masiva, abierta y gratuita por medio de una metodología basada en la videosimulación y en el anhelo del trabajo colaborativo del estudiante.

De acuerdo con Castaño \& Cabero (2013, p. 89), los cursos MOOC presentan una serie de características que les diferencian de los cursos tradicionales de la educación on line:

- «Es un recurso educativo que tiene cierta semejanza con una clase, con un aula.

- Con fechas de comienzo y finalización.

- Cuenta con mecanismos de evaluación.

- Es online.

- De uso gratuito.

- Es abierto a través de la web, y no tiene criterios de admisión.

- $\quad$ Permite la participación interactiva a gran escala de cientos de estudiantes.»

En realidad desde la aparición de los primeros cursos MOOC se han ido generando nuevas versiones de estos cursos, de forma que nos podemos encontrar con cursos denominados MOOC que no se ajustan del todo a las primeras características iniciales de los cursos MOOC, ya que los primeros MOOC, denominados cMOOC, estaban más comprometidos con el aprendizaje abierto y la conectividad. En la actualidad los cursos MOOC más aplicados a nivel educativo son los denominados xMOOC. Son los cursos donde mayor número de matrículas se realizan

$$
-140 \text { - }
$$


y también son los más utilizados por los docentes universitarios. En los cursos xMOOC los contenidos se presentan de una forma organizada donde aparecen una serie de pruebas automatizadas y poseen una gran difusión mediática (Rodríguez, 2012). Están basados en la adquisición de contenidos y se basan en un modelo de evaluación muy parecido a las clases tradicionales (con unas pruebas más estandarizadas y concretas) (Martí, 2012). Los XMOOC se basan en una larga serie de métodos tradicionales a distancia. Algunos permiten la colaboración a través de foros de discusión o una evaluación entre pares, este es una sistema que fue implementado por Coursera» (Bartolome \& Steffens, 2015).

Este tipo de MOOC tiene las características básicas del aprendizaje tradicional de la enseñanza en línea (e-learning), pero con una serie de adaptaciones para que se desarrolle en un formato MOOC.

A través de estos cursos se promueve la autoorganización, la conectividad, la diversidad y el control descentralizado de los procesos de enseñanza-aprendizaje. Otras modalidades a tener en cuenta dentro del universo MOOC son aquellas que hacen referencia a cursos con características aún más específicas, surgidas de los xMOOC .Una de ellas son los cursos LOOC (Little Open Online Course), que Kolowich (2012) nos define como pequeños cursos MOOC con menos de unos cientos de estudiantes. Por otro lado Rivard (2013) define los SPOC «Small Private Online Course», como cursos con pocos estudiantes que son de carácter semiprivado. Más aún, promueven la admisión de una cohorte determinada para promover la interacción alumno-alumno y mejorar los resultados de aprendizaje (Chauhan, 2014).
MacVie, (2013) señala que un «Small Private Online Course», se diferencia de un MOOC principalmente porque no es abierto, es decir, cualquier persona no puede realizarlo ya que se necesitan unos requisitos específicos para poder participar en él. Aún así también utiliza las siglas SPOC para hacer referencia a «Self Pace Online Course» esto son un tipo determinado de cursos que siempre están disponibles, de forma que cualquier persona en cualquier momento y en cualquier lugar puede comenzar a realizarlos, marcándose sus propias pautas y ritmos. (MacVie, 2013).

Estas siglas dan mucho de sí y podemos hablar de una tercera variante SPOC que serian los «Small Private Open Courses» en este caso nos encontramos con que el termino Open hace referencia por un lado a que cualquier persona puede acceder en cualquier momento y lugar a los materiales que son ofrecidos bajo una licencia que lo permite, pero también este tipo de cursos, se abren periódicamente a pequeños grupos con los que los creadores del curso y sus contenidos interactúan directamente con los participantes. Son cursos donde el profesor es algo más cercano y directo. (Mac Vie, 2013).

En los cursos SPOC la metodología utilizada en sus diferentes variedades se basa totalmente en la filosofía MOOC y los docentes tienden a utilizar o reutilizar materiales que ya disponen de otros cursos y ajustarlos a un formato más pequeño en lo relativo a participantes.

Hay autores que argumentan que los SPOC son una vía para dar respuesta a algunas de las principales críticas que están recibiendo los MOOC (Fox, 2013).

Los estudios realizados sobre estos cursos nos muestran que el éxito de los estudiantes que los realizan es importante y en sus evaluaciones, achacan a el papel

$-141-$

Píxel-Bit. Revista de Medios y Educación. $N^{o} 52$ Enero 2018. ISSN: 1133-8482. PIXEL e-ISSN: 2171-7966. doi: http://dx.doi.org/10.12795/pixelbit.2018.i52.10 
desempeñado por el profesor de refuerzo, apoyo y atención el haber logrado esos buenos resultados (Oremus, 2013).Por lo tanto los cursos SPOC se ven como algo más cuidado y cercano para el estudiante y el profesor (Fox, 2013). La inserción de los cursos MOOC en general en sus diferentes variantes cada vez está tomando más importancia dentro de la investigación educativa, autores como Dillenbourg, Fox, Claude Kirchner, Mitchell \& Wirsing (2014) destacan la importancia de realizar estudios sobre la inserción de estos cursos en la formación universitaria, ya que pueden ser un buen recurso tanto para el profesorado como para el alumnado. Por otra parte, la revisión de la literatura nos indica la importancia de atender al punto de vista de los alumnos en la investigación sobre MOOC (Perna et al, 2014; Wiebe, Thompson y Beherend, 2015), recomendación que hacemos nuestra en este artículo.

\section{Metodología}

A través de este artículo se analiza la utilización de los cursos MOOC dentro de la universidad, como un posible recurso a utilizar. En concreto, se pretende investigar la aplicación de un curso SPOC en una asignatura de segundo grado. Para ello se ha diseñado e implantado un curso SPOC que fue realizado de forma on line por parte del alumnado de la asignatura, mientras que el mismo curso, fue realizado en el contexto del aula presencial. De esta forma lo que se intentaba era realizar una comparativa de resultados entre los dos grupos de estudio.

\subsection{Objetivo}

El objetivo principal es valorar la eficacia de insertar nuevos formatos pedagógicos originarios de los cursos MOOC dentro del contexto universitario como complemento a asignaturas de grado.

\subsection{Hipótesis}

Las hipótesis planteadas en esta investigación han sido las siguientes:

H1.La motivación para el aprendizaje autorregulado es superior en aquellos alumnos que han realizado la formación online.

$\mathrm{H} 2$.El alumnado que realiza el curso online otorga valoraciones superiores al curso en cuanto aprendizaje frente a los que realizan el curso de forma presencial.

H3. El alumnado que realiza el curso online presenta un mayor entusiasmo y atención hacia el mismo frente a los que realizan el curso de forma presencial.

H4.El curso online obtiene mejores valoraciones en cuanto a la organización que el presencial.

H5.La opinión sobre interacción grupal es más positiva en el alumnado que ha realizado el curso online frente a los que lo han desarrollado de forma presencial.

H6.La actitud del profesorado es mejor valorada por los que realizan el curso online frente a los que lo realizan de forma presencial.

H7.Los estudiantes que realizan el curso online valoran de forma más positiva el sistema de evaluación que los que realizan el curso de forma presencial.

H8. El alumnado que realiza el curso on line, obtienen mejores calificaciones que los del grupo control.

-142 - 


\begin{tabular}{|c|c|c|c|c|c|c|c|c|c|c|}
\hline \multirow{5}{*}{$\begin{array}{l}\text { Creo que la } \\
\text { formación on line es un } \\
\text { buen complemento para } \\
\text { la asignatura }\end{array}$} & & \multirow{2}{*}{\multicolumn{2}{|c|}{ Experimental }} & \multirow{2}{*}{\multicolumn{2}{|c|}{$\begin{array}{l}\text { Grupo } \\
\text { Control }\end{array}$}} & \multirow{2}{*}{\multicolumn{2}{|c|}{ Total }} & \multirow{2}{*}{$\begin{array}{c}\text { Chi } \\
\text { cuadrado }\end{array}$} & \multirow[b]{2}{*}{ gl } & \multirow[b]{2}{*}{ Sig. } \\
\hline & & & & $\mathrm{N}^{\mathrm{C}}$ & & & & & & \\
\hline & Sí & 252 & $87.2 \%$ & 17 & $89.5 \%$ & 269 & $87,3 \%$ & & & \\
\hline & No & 37 & $12,8 \%$ & 2 & $10,5 \%$ & 39 & $12,7 \%$ & 0,084 & 1 & 0.773 \\
\hline & Total & 289 & $100,0 \%$ & 19 & $100,0 \%$ & 308 & $100,0 \%$ & & & \\
\hline Creo que la formación & Si & 269 & $93,1^{\circ}$ & 17 & $89,5 \%$ & 286 & $92,9 \%$ & & & \\
\hline $\begin{array}{l}\text { on line es una buena } \\
\text { opción para la } \\
\text { formación del }\end{array}$ & No & 20 & $6,9 \%$ & 2 & $10,5 \%$ & 22 & $7,1 \%$ & 0,349 & 1 & 0,554 \\
\hline profesorado & Total & 289 & $100,0 \%$ & 19 & $100,0 \%$ & 308 & $100,0 \%$ & & & \\
\hline
\end{tabular}

Tabla: Hipótesis 1.

\subsection{Muestra}

La muestra estaba compuesta por estudiantes universitarios de la Universidad Pública Vasca/ Euskal Herriko Unibertsitaea UPV/EHU en concreto, alumnado de la escuela de Magisterio de Bilbao, situada en el Campus de Leioa (Bizkaia). Todos los participantes formaban parte del segundo curso de los grados que se ofertan en ese centro, Grado de Educación Primaria y Grado de Educación Infantil. El número total de alumnado que participó en la investigación fue de 308 alumnos, de los cuales el $23,1 \%$ fueron hombres y el 76,9\% eran mujeres.

La muestra fue dividida en dos grupos, grupo control y grupo experimental. El grupo control realizó el curso de forma presencial mientras que el grupo experimental lo realizó de forma virtual, a través de un curso SPOC.

\subsection{Instrumentos Utilizados}

Para realizar el estudio se utilizó el cuestionario SEEQ (Students' Evaluation of Educational Quality), este cuestionario es un instrumento de valoración y mejora de la enseñanza, que permite analizar la eficacia de la enseñanza y a su vez destacar o detectar cuáles deben ser los puntos de mejora que debemos tener en cuenta. El SEEQ fue diseñado por el profesor Herbert Marsh a finales de la década de 1970 y permite analizar la eficacia de la enseñanza utilizando una serie de factores, cada uno de los cuales, consta de varios ítems, que son valorados en una escala tipo Likert de cinco opciones (muy en desacuerdo, en desacuerdo, ni de acuerdo ,ni en desacuerdo, de acuerdo y muy de acuerdo) y que pueden ser utilizados, como ya se ha comentado, con finalidad formativa y un claro objetivo de iniciar una mejora continuada del proceso docente. El curso SPOC fue insertado en una plataforma denominada MetaMOOC que permitió realizar al profesorado diferentes actividades y mantener con el alumnado una relación directa a través de un foro que fomentaba la colaboración para el desarrollo del curso SPOC.

\section{Resultados}

El análisis de resultados se ha llevado a cabo a través del programa estadístico informático IBM SPSS. Los datos se presentan en base a las diferentes hipótesis trabajadas en el estudio. En relación a la Hipótesis 1.La motivación para el aprendizaje autorregulado es superior en aquellos alumnos que han realizado la formación online. Debemos manifestar que no se dan diferencias entre ambos grupos por lo tanto las puntuaciones, entre el grupo experimental

$-143-$

Píxel-Bit. Revista de Medios y Educación. $N^{o} 52$ Enero 2018. ISSN: 1133-8482. e-ISSN: 2171-7966. doi: http://dx.doi.org/10.12795/pixelbit.2018.i52.10 


\begin{tabular}{|c|c|c|c|c|c|c|c|c|c|c|c|c|}
\hline \multirow[b]{4}{*}{ Aprendizaje } & \multicolumn{8}{|c|}{ Grupo } & \multicolumn{3}{|c|}{$\mathrm{U}$ de } & \multirow{3}{*}{$\begin{array}{c}\text { Sig. } \\
\text { Asintót. } \\
\text { (bilateral) }\end{array}$} \\
\hline & & perimer & & & Control & & & Total & & Mann- & Z & \\
\hline & $\mathrm{N}$ & Media & D.T. & $\mathrm{N}$ & Media & D.T. & $\mathrm{N}$ & Media & D.T. & Whitney & & \\
\hline & 289 & 3,78 & 0,77 & 19 & 4,39 & 0,44 & 308 & 3,81 & 0,77 & 1325,500 & 43230,500 & 0,000 \\
\hline
\end{tabular}

Tabla 2: Hipótesis 2.

\begin{tabular}{|c|c|c|c|c|c|c|c|c|c|c|c|c|}
\hline \multirow[b]{4}{*}{ Entusiasmo } & \multirow{2}{*}{\multicolumn{3}{|c|}{ Experimental }} & \multirow{2}{*}{\multicolumn{3}{|c|}{$\begin{array}{l}\text { Grupo } \\
\text { Control }\end{array}$}} & \multicolumn{5}{|c|}{ U de } & \multirow{3}{*}{$\begin{array}{c}\text { Sig. } \\
\text { asintót. } \\
\text { (bilateral) }\end{array}$} \\
\hline & & & & & & & & Total & & Mann- & $Z$ & \\
\hline & $\mathrm{N}$ & Media & D.T. & $\mathrm{N}$ & Media & D.T. & $\mathrm{N}$ & Media & D.T. & Whitney & & \\
\hline & 289 & 3,46 & 0,87 & 19 & 4,28 & 0,49 & 308 & 3,51 & 0,87 & 1147,500 & 43052,500 & 0,000 \\
\hline
\end{tabular}

Tabla 3: Hipótesis 3.

\begin{tabular}{cllcccc}
\hline & & Grupo & & Ude & Sig. \\
Experimental & Control & Total & Mann- & Z & Asintót. \\
(bilateral)
\end{tabular}

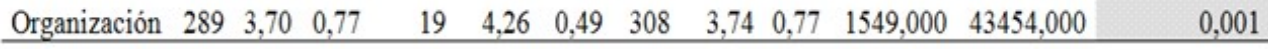

Tabla 4: Hipótesis 4.

\begin{tabular}{|c|c|c|c|c|c|c|c|c|c|c|c|c|}
\hline & Ex & $\begin{array}{l}\text { perimen } \\
\text { Media }\end{array}$ & $\begin{array}{l}\text { atal } \\
\text { D.T. }\end{array}$ & $N$ & $\begin{array}{l}\text { Grupo } \\
\text { Control } \\
\text { Media }\end{array}$ & D.T. & $N$ & $\begin{array}{c}\text { Total } \\
\text { Media }\end{array}$ & D.T. & $\begin{array}{c}\text { U de } \\
\text { Mann- } \\
\text { Whitney }\end{array}$ & $z$ & $\begin{array}{c}\text { Sig. } \\
\text { Asintót. } \\
\text { (bilateral) }\end{array}$ \\
\hline grupal & 289 & 3,53 & 0,65 & 19 & 3,39 & 0,72 & 308 & 3,52 & 0,66 & 2397,000 & 2587,000 & 0,353 \\
\hline
\end{tabular}

Tabla 5: Hipótesis 5.

\begin{tabular}{|c|c|c|c|c|c|c|c|c|c|c|c|c|}
\hline \multirow[b]{3}{*}{ Actitud } & \multicolumn{7}{|c|}{ Grupo } & Total & \multicolumn{2}{|r|}{$\mathrm{Ude}$} & Z & \multirow{2}{*}{$\begin{array}{c}\text { Sig. } \\
\text { asintót. } \\
\text { (bilateral) }\end{array}$} \\
\hline & $\mathrm{N}$ & Media & D.T. & $\mathrm{N}$ & Media & D.T. & $\mathrm{N}$ & Media & D.T. & Whitney & & \\
\hline & 289 & 3,65 & 0,97 & 19 & 4,66 & 0,58 & 308 & 3,71 & 0,98 & 1049,500 & 42954,500 & 0,000 \\
\hline
\end{tabular}

Tabla 6: Hipótesis 6.

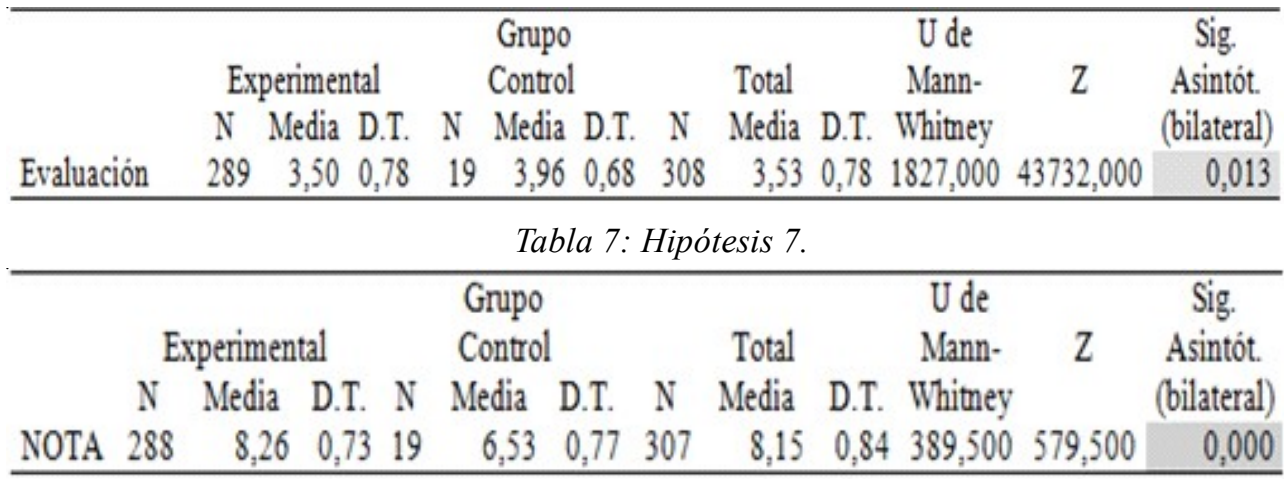

Tabla 8: Hipótesis 8.

- 144 -

Píxel-Bit. Revista de Medios y Educación. No 52 Enero 2018. ISSN: 1133-8482.

e-ISSN: 2171-7966. doi: http://dx.doi.org/10.12795/pixelbit.2018.i52.10 
y el grupo control son muy similares. (véase ,tabla 1).

La autorregulación del aprendizaje por el estudiante en los entornos virtuales es fundamental, ya que facilita pasar de su percepción como herramientas tecnológicas, a su utilización como herramientas pedagógicas. Las competencias que necesita el alumnado universitario son aquellas que le permiten ser autónomo a la hora de enfrentarse a las demandas de los nuevos aprendizajes que asumirá a lo largo de su vida. Es significativo que en este caso son los componentes del grupo control quienes manifiestan tener más capacidades de motivación para el aprendizaje autónomo. Ambos grupos consideran que la formación on line es un buen complemento para la asignatura y a su vez también consideran que la formación on line es una buena opción para la formación del profesorado

En cuanto a la Hipótesis 2.El alumnado que realiza el curso online otorga valoraciones superiores al curso en cuanto aprendizaje frente a los que realizan el curso de forma presencial. No se válida la hipótesis (véase Tabla 2).Es el grupo control quien obtiene valoraciones superiores en satisfacción hacia el aprendizaje a través de los datos obtenidos con el cuestionario (SEEQ) (Students' Evaluation of Educational Quality).

Investigaciones realizadas como la de (Kuzmanovic, Savic, Gusavac, MakajicNikolic, \& Panic, 2013) señalan que los indicadores de una buena docencia para el alumnado son: claridad en las presentaciones; enfoque metodológico y sistemático; disponibilidad del profesorado; información a los alumnos sobre el trabajo de la asignatura; potenciación de la participación en clase y consideración de las preguntas de los alumnos. Un estudio de Sun, Tsai, Finger,
Chen y Yeh (2008) reveló siete factores críticos que afectan a la satisfacción percibida por los estudiantes en e-Learning: la ansiedad ante el ordenador del estudiante, la actitud del tutor de e-Learning, la flexibilidad del curso, la calidad del curso, la utilidad percibida, la facilidad de uso percibida, y la diversidad en las evaluaciones. A través de los datos obtenidos vemos que todo esto ha sido más valorado en el curso presencial dentro del contexto del aula.

Los datos referentes a la Hipótesis 3. El alumnado que realiza el curso online presenta un mayor entusiasmo y atención hacia el mismo frente a los que realizan el curso de forma presencial. , manifiestan (véase Tabla 3 ), muestran que es el grupo control quien obtiene valoraciones superiores en entusiasmo frente la puntuación, en entusiasmo que manifiestan los participantes del grupo experimental en el test (SEEQ) (Students' Evaluation of Educational Quality).

El entusiasmo está ligado a la motivación y los datos obtenidos revelan que ha sido el grupo control quien ha manifestado mayor nivel de entusiasmo. Algo fundamental a la hora de llevar a cabo el proceso de enseñanzaaprendizaje. El profesorado motiva más y mejor a aquellos/as alumnos/as que se consideran sujetos activos de lo que está sucediendo en el aula. Los datos obtenidos muestran que en el curso on line, a pesar de que su nivel de entusiasmo no es bajo, el alumnado se ha sentido menos motivado que en el curso presencial.

Los datos de la Hipótesis 4.El curso online obtiene mejores valoraciones en cuanto a la organización que el presencial. , expresan que la hipótesis planteada no se valida (véase Tabla 4).

El grupo control obtiene valoraciones superiores en organización a través de los

-145 -

Píxel-Bit. Revista de Medios y Educación. No 52 Enero 2018. ISSN: 1133-8482. PIXEL e-ISSN: 2171-7966. doi: http://dx.doi.org/10.12795/pixelbit.2018.i52.10 
datos obtenidos del test (SEEQ) (Students' Evaluation of Educational Quality). Podemos definir la organización, como la forma en que se articulan los distintos componentes del curso y de ella depende su coherencia. Los aspectos fundamentales que recoge este factor a través de sus ítems son la claridad de las explicaciones del docente y la adecuación en la programación del tema tratado. De la forma en la que el docente desarrolla la docencia y fundamente las ideas y los conceptos depende en gran medida el nivel de comprensión del discente. En esta ocasión, es el alumnado del curso presencial quienes han valorado mejor la organización del curso. Eso no quiere decir que los participantes del grupo experimental refieran una mala organización en el curso realizado.

Los datos extraídos en la Hipótesis 5.La opinión sobre interacción grupal es más positiva en el alumnado que ha realizado el curso online frente a los que lo han desarrollado de forma presencial, exponen que no hay diferencias entre los grupos (véase Tabla 5), señalan que ambos grupos consideran que la interacción grupal es adecuada.

La interacción grupal refuerza el conocimiento y el aprendizaje dentro de los grupos y en este caso ambos grupos manifiestan el mismo nivel de interacción grupal. La ANECA (2003) incluye entre las competencias básicas de la educación superior, la importancia del trabajo colaborativo en el proceso de aprendizaje y se incluye también en el actual marco de convergencia europeo. El apoyo entre iguales tienen un impacto significativo en la utilidad percibida del e-Learning para el aprendizaje individual, (Cheng, Wang, Moormann, Olaniran, \& Chen, 2012). Niveles más altos de conocimiento retenido y el apoyo al compañero de trabajo incrementan la transferencia del aprendizaje (Homklin, Takahashi, \& Techakanont, 2014). El rol del docente en las dos modalidades de enseñanza ha estado orientado hacia la mediación como tutor y gestor del aprendizaje.

En cuanto a la Hipótesis 6. La actitud del profesorado es mejor valorada por los que realizan el curso online frente a los que lo realizan de forma presencial, los datos (véase Tabla 6) los datos extraídos muestran que es el grupo control quien valora más al profesor frente a la valoración que algo más baja, que da el grupo experimental.

El grupo control obtiene valoraciones superiores en actitud del profesor en los resultados obtenidos a través del (SEEQ) (Students' Evaluation of Educational Quality). Los docentes en su práctica profesional no sólo deben dominar los contenidos que imparten sino que es necesario que faciliten el aprendizaje y la participación de todos los estudiantes, aunque en los dos grupos que han participado en la investigación la valoración del profesor no ha sido negativa, es en el grupo control donde se da una valoración más elevada al trabajo realizado. Las necesidades no cubiertas al final de la formación en los estudiantes, se relacionan con: la atención por parte de los docentes, el filtro de los mensajes en los foros, la calidad de los materiales didácticos o los recursos disponibles, entre otras. En ocasiones, parte del alumnado on line realiza una mayor demanda al profesor de funciones de apoyo, orientación y motivación desde un correcto uso docente de las herramientas de comunicación de la plataforma (Santoveña, 2007), ya que está acostumbrado al formato presencial. Un aspecto en el que coinciden en investigación hoy en día y que viene a dar sentido global a la calidad de la formación

- $146-$

Píxel-Bit. Revista de Medios y Educación. No 52 Enero 2018. ISSN: 1133-8482. e-ISSN: 2171-7966. doi: http://dx.doi.org/10.12795/pixelbit.2018.i52.10 
online es la implicación de los estudiantes en todas las actividades formativas (GarcíaValcárcel \& Tejedor, 2011).

A continuación (véase Tabla 7) se muestran los datos referentes a la Hipótesis 7.Los estudiantes que realizan el curso online valoran de forma más positiva el sistema de evaluación que los que realizan el curso de forma presencial.

El grupo control obtiene valoraciones superiores en Evaluación en los resultados obtenidos a través del (SEEQ) (Students' Evaluation of Educational Quality) por lo que la hipótesis planteada no se valida. El sistema de evaluación aplicado a los grupos fue el mismo, pero el grupo control lo valora de forma más positiva, aunque el grupo experimental no aporta una valoración negativa del método utilizado. Zapata (2012) señala que «los instrumentos y las prácticas de la evaluación deben hacer énfasis, integrando indicadores si se ha desarrollado un diseño tecnológico e instruccional que integre las filosofías de compartir y adicionar».

Para finalizar a continuación se muestran los datos pertenecientes a la Hipótesis 8. El alumnado que realiza el curso on line, obtienen mejores calificaciones que las del grupo control. En esta ocasión es significativo que es el grupo experimental quien ha obtenido calificaciones superiores a las del grupo control.

Podemos afirmar que a través de la utilización de una metodología diferente, como es el formato utilizado en la educación on line por los cursos MOOC, se ha podido desarrollar el aprendizaje activo y autónomo y mejorar el análisis crítico, la reflexión, las calificaciones, la motivación y el entusiasmo por la asignatura (Svinicki \& MacKeachie, 2014; Moraros et al, 2015), aunque diferentes variables mencionadas han tenido puntuaciones más altas en la metodología presencial. Finalmente el alumnado se ha mostrado activos frente al aprendizaje y han adquirido competencias y capacidades que les permitan avanzar en su desarrollo educativo.

\section{Conclusiones}

El objetivo principal de esta investigación ha sido valorar la eficacia de insertar nuevos formatos pedagógicos originarios de los cursos MOOC dentro del contexto universitario como complemento a asignaturas de grado. A través de los datos obtenidos podemos manifestar que las dos experiencias de aprendizaje desarrolladas dentro de una asignatura de grado han sido valoradas de forma muy positiva, es decir tanto la experiencia realizada de forma presencial, como la experiencia realizada a través del curso SPOC. Las puntuaciones obtenidas por el grupo experimental, que realizó el curso SPOC, fueron buenas en todos los ítems, destacando sobretodo la nota obtenida ya que ha sido más alta que las obtenidas en el grupo control. Por lo tanto a través de los datos analizados podríamos manifestar que la utilización de los curso SPOC como complemento a las asignaturas de grado es algo positivo .Debemos destacar la importancia que tiene la realización de investigaciones con este tipo de formatos pedagógicos o similares como son, las aulas invertidas o la utilización de los cursos MOOC como material de estudio (MOOCs as books), ya que aportan más información sobre la riqueza de este tipo de recursos y el beneficio de los mismos tanto para el alumnado como para el profesorado universitario. 


\section{Referencias bibliográficas.}

Aguaded, J. I., Vázquez-Cano, E. \& Sevillano, M. L. (2013). MOOCs, ¿turbocapitalismo de redes o altruismo educativo? En «SCOPEO INFORME Núm. 2: MOOC: Estado de la situación actual, posibilidades, retos y futuro» (pp. 74-90). Salamanca: Universidad de Salamanca Servicio de Innovación y Producción Digital. Disponible en: http://scopeo.usal.es/wpcontent/uploads/2013/06/scopeoi002.pdf.

ANECA (2003). El Programa de Convergencia Europea de Aneca. (2003-2006). Agencia Nacional de Evaluación de la Calidad y Acreditación. Consultado [16/01/2016].

Castaño, C. \& Cabero, J. (2013). Enseñar y aprender en entornos m-learning. Madrid: Síntesis. Consultado [29/03/2015].

Bartolomé, A\& Steffens, K (2015). ¿Son los MOOC una alternativa de aprendizaje? Comunicar, $n^{\circ} 44$, v. XXII, 2015 | Revista Científica de Educomunicación | Consultado [27/07/2015].

Chauhan, A. (2014). Massive Open Online Courses (MOOCS): Emerging Trends in Assessment and Accreditation. Digital Education Review. № 25, http://greav.ub.edu/ der/

Cheng, M., Wang, J., Moormann, B.A., Olaniran, N. (2012). The effects of organizational learning environment factors on e-learning acceptance. Computers \& Education, 58 (3) (2012), pp. 885-899

Fox, A (2013). From MOOC to SPOCs. Communications of the ACM, 56(12), pp. 3840. :http://cacm.acm.org/magazines/2013/12/ 169931 -from-MOOC-to-spocs/fulltext. Consultado [09/07/2015].

Dillenbourg, P., Fox, A., Kirchner, C., Mitchell, J. y Wirsing, M. (2014): Massive Open Online Courses: Current state and
Perspectives. Dagstuhl Manifestos, Vol.4, Issue 1, pp. 1-27 Disponible en doi: 10.4230/ DagMar.4.1.1.

García-Valcárcel, A. \& Tejedor, F. J. (2011). Variables TIC vinculadas a la generación de nuevos escenarios de aprendizaje en la enseñanza universitaria. Aportes de las curvas ROC para el análisis de diferencias. Educación XX1, 14(2), 43-78. Consultado [05/ 01/2016].

Ghemawat, P. (2017). Strategies for Higher Education in the Digital Age. California Management Review. 59 (4), 56-78. https:// doi.org/10.1177/0008125617717706

Homklin, T., Takahashi, Y., \& Techakanont, K. (2014). The influence of social and organizational support on transfer of training: evidence from Thailand. International Journal of Training and Development, 18(2), 116-131. doi:10.1111/ijtd.12031. Consultado [12/03/ 2016].

Kolowich, S. (2012). Inside Higher Ed. «MOOC' Little Brother.» From the Internet: http://www.insidehighered.com/news/2012/ 09/06/u-maine-campus-experiments-smallscalehigh- touch-open-courses. Consultado [10/10/2015].

Kuzmanovic, M., Savic, G., Gusavac, B. A., Makajic-Nikolic, D., \& Panic, B. (2013). A

Conointbased approach to student evaluations of teaching performance.Expert Systems with Applications 40, 4083-4089. Consultado [12/06/2015].

MacVie, L. (2013). SPOCS: The Doable Open Courses for Medium-Sized Institutions. Leah MacVie. Recuperado a partir de http:// leahmacvie.com/2013/12/spocs-are-doable/

Méndez García, C.M. (2013). «Diseño e implementación de cursos abiertos masivos en línea (MOOC): expectativas y consideraciones prácticas». En Revista de Educación a Distancia (RED). Murcia:

$$
\text { - } 148 \text { - }
$$


Universidad de Murcia, nº39. Consultado [12/ 10/2015].

Oremus, W. (2013, septiembre 18). Forget MOOCs.Freeon line classrooms shouldn't replace teachers and classroms. The should make them better. Slate. Recuperado a partir de http://www.slate.com/articles/technology/ $\mathrm{t}$ e c h n o l o g y / $201013 / 0$ / spocs_small_private_online_clas ses may be better than moocs.html.

Rivard, R. (2013). Inside Higher Ed, «Reins on Moonlighting.» From the Internet: http:// www.insidehighered.com/news/2013/05/08/ u-pennsylvania-drafts-guidelineskeepprofessors- competing-against-it-online. Consultado [17/09/2015].

Rodríguez, O. (2012). MOOC and the AIStanford like Courses:Two Successful and Distinct Course Formats for Massive Open Online Courses. European Journal of Open, Distance and E Learning, 2. (http://goo.gl/ 0U29S). Consultado [27/07/2015].

Santoveña, S. M. (2007). Análisis de cursos de Educación Social en entornos virtuales de aprendizaje y su influencia en la calidad. Enseñanza, 25, 77-90. Consultado [25/02/ 2016].

Pei-Chen Sun, Ray J. Tsai, Glenn Finger, Yueh-Yang Chen, \& Dowming Yeh (2008).What drives a successful e-Learning? An empirical investigation of the critical factors influencing learner satisfaction.Computers \& Education.50 (2008) 1183-1202. Available on line at www.sciencedirect.com.

Perna, L.W. et al. (2014). Moving Through MOOCs. Understanding the Progression of Users in Massive Open Online Courses. Educational Researcher, Vol. 43 No. 9, pp. 421432. 0013189X14562423
UNESCO (2012).Declaración de París sobre los Recursos Educativos Abiertos (Junio 2012). http://www.unesco.org/new/ fileadmin/multimedia/hq/ci/wpfd2009/ spanish_declaration.html.

Vázquez-Cano, E. (2013). El videoartículo: nuevo formato de divulgación en revistas científicas y su integración en Moocs. Comunicar, 41, 83-91. DOI: 10.3916/C41-201308 .

Vizoso Martín, C.Mª (2013). ¿Serán los COMA (MOOC), el futuro del e-learning y el punto de inflexión del sistema educativo actual? Revista Didáctica, Innovación y Multimedia, 25. Disponible en: http:// www.pangea.org/dim/revista25.

Wiebe, E., Thompson, I. y Beherend, T. (2015). MOOCs From the Viewpoint of the Learner. A Response to Perna et al. (2014). Educational Researcher, Vol. 44 No. 4, pp. 252254. 0013189X15584774 https://doi.org/10.3102/

Zapata -Ros, M (2014). Los MOOC en la crisis de la Educación Universitaria. Docencia, diseño y aprendizaje: Un modelo previsible. (http://www.um.es/ead/mzapata/). Consultado [18/07/2015].

Fecha de recepción: 21-02-2017

Fecha de evaluación: 22-02-2017

Fecha de aceptación: 22-02-2017

- 149 -

Píxel-Bit. Revista de Medios y Educación. No 52 Enero 2018. ISSN: 1133-8482. e-ISSN: 2171-7966. doi: http://dx.doi.org/10.12795/pixelbit.2018.i52.10 